\title{
Understanding Complex Environments with the Feedforward Torch
}

\author{
Jo Vermeulen, Kris Luyten, and Karin Coninx \\ Hasselt University - tUL - IBBT, \\ Expertise Centre for Digital Media, \\ Wetenschapspark 2, B-3590 Diepenbeek, Belgium. \\ [jo.vermeulen, kris.luyten, karin. coninx] @uhasselt. be
}

\begin{abstract}
In contrast with design flaws that occur in user interfaces, design flaws in physical spaces have a much higher cost and impact. Software is in fact fairly easy to change and update in contrast with legacy physical constructions where updating their physical appearance is often not an option. We present the Feedforward Torch, a mobile projection system that targets the augmentation of legacy hardware with feedforward information. Feedforward explains users what the results of their action will be, and can thus be seen as the opposite of feedback. A first user study suggests that providing feedforward in these environments could improve their usability.
\end{abstract}

Keywords: feedforward, intelligibility, mobile projection, legacy systems

\section{Introduction}

Creating new usable ubicomp systems, especially systems that support walk-upand-use scenario's, is covered in various facets in literature. While most literature discusses newly created systems and setups, we are interested in systems that are already present in our environment and are meant to be used over longer periods (e.g., 10 years and beyond). We start from our existing environments in which we reside on a daily basis. Our environment exposes many automated or computerized systems that were meant to be walk-up-and-use systems. These systems might not be context-aware or smart (i.e., are not pro-active nor use sensors for interaction), but their physical designs are tightly integrated in our environment and their usage is often part of our daily routines.

Fig. 1 shows three examples of legacy systems one can find in various public buildings. Fig. 1(a) shows a button that appears to trigger the power socket below for a predefined period of time. Nevertheless, using this button will also turn on the television and VCR in the same classroom. Fig. 1(b) presents three boxes that need to be controlled in case one detects fire. The left-most button's

\footnotetext{
${ }^{0}$ The original publication is available at www.springerlink.com at http://link. springer.com/chapter/10.1007/978-3-642-34898-3_22
} 
function is clear, but what do users have to do with the two others? Do you need a key? Is it safe to turn a key when it says "evacuate" below? What will happen in this case? Finally, Fig. 1(c) shows a terminal for recharging a contactless payment card using Near-Frequency Communication (NFC). Two cards need to be used: the contactless payment card and a debit card to transfer money from on to another. The sequence of steps and when to use each card is often a source of confusion. These are just three legacy systems that we found in the wild. While we were developing the Feedforward Torch, we collected many more examples of legacy systems that were designed for walk-up-and-use scenarios but cause problems for users as it is not immediately clear how to interact with them.

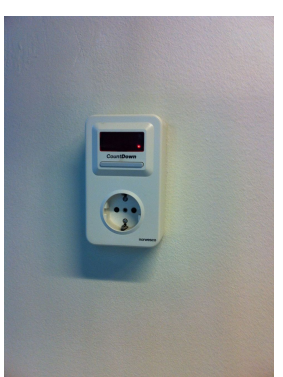

(a) Mysterious button

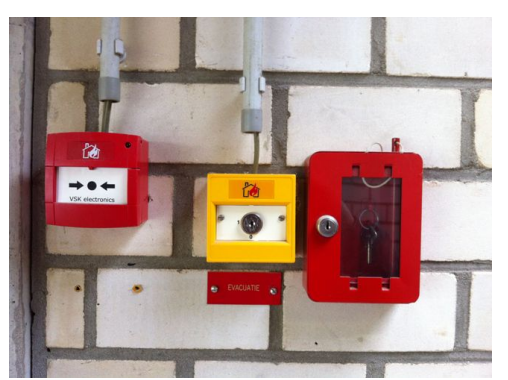

(b) Confusing fire alarm

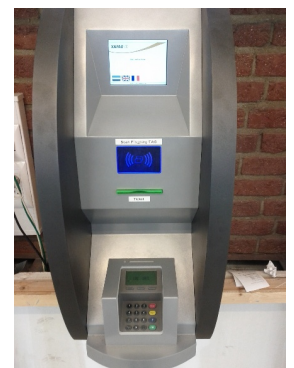

(c) NFC recharging terminal

Fig. 1. Three examples of legacy systems that have design flaws that make them unsuitable for walk-up-and-use usage. All systems presented come from the same university building. People that frequent this environment tend to have difficulties using these systems because of unclear or missing feedforward information.

Due to the fact that the designs of these systems do not convey how they work, users have difficulties predicting the behaviour and the available features of the system. Moreover, it is often not clear to users how they can interact with these systems. For context-aware systems, Bellotti and Edwards [2] have proposed the concept of intelligibility. Intelligible systems have built-in support for helping users understand how they work. Intelligibility is an important feature for context-aware systems that take actions on the user's behalf, and rely on implicit input and complex inferencing. For non-smart systems, we tend to rely on "good design" to make sure users know how to interact with them. However, our environment is often comprised of a combination of legacy and new systems, due to budgetary considerations. While each of these systems might have been well-designed, their designers have often not considered these systems to be combined together. Since a combination of legacy and new systems tends to be seen as a temporary solution, it would often be too costly to rethink the entire physical design so that it better matches user's expectations. For this reason, we 
hypothesize that legacy systems (or combinations of legacy and newer systems) that cannot be altered, could also benefit from intelligibility to help users learn how to interact with them.

Our focus for augmenting the legacy systems is on feedforward, a specific type of intelligibility that tells users what will happen when they perform a certain action. Feedforward informs the user about what the result of an action will be, and can thus be seen as the opposite of feedback. Well-designed feedforward is an effective tool for bridging Norman's Gulf of Execution [4] - the gap between a user's goals for action and the means for executing those goals. Feedforward has been successfully applied in gesture-based interaction to help users learn, perform and remember gestures [1]. Additionally, Lim and Dey's "What if?"-questions [3] can be seen as a type of feedforward for context-aware systems. For physical interfaces, feedforward is often conveyed by the design and form-factor of the interface. However, if the design fails to convey feedforward information, this is very cumbersome and expensive to fix after deployment. Physically changing the interface design to include better feedforward would imply that every instance of the system needs to be fixed separately. For example, we found the fire alarm interface (shown in Fig. 1(b)) over 50 times in the same building.

In this paper, we present the Feedforward Torch, a combination of a mobile phone and mobile projector that provides feedforward information about different objects in their physical environment. Our solution augments the objects, more specifically legacy systems, during usage and does not require physical changes. We have currently built a prototype of the system and conducted a user study to investigate the suitability of this technique as a way to overcome design flaws of legacy systems.

\section{Related Work}

The possibility of augmenting physical environments using mobile projectors was first demonstrated by Raskar et al. with their iLamps [8] project. Earlier work [6] focused on steerable, ceiling-mounted projectors. Later, Raskar et al. extended these mobile projectors with RFID readers and photosensing capabilities to identify the physical objects that were being augmented [7]. In recent years, advances in hardware have enabled compact prototypes that can be embedded into smartphones, and different interaction possibilities have emerged [10].

The Feedforward Torch (see Sect. 3) is inspired by existing work on portable projectors. However, our contribution lies not in producing high-quality graphics on projected surfaces, or in interaction techniques. We rather explored how this setup can be used as a ubiquitous guidance system that helps users deal with legacy systems that suffer from design flaws.

Previously, Vermeulen et al. [11] investigated the use of steerable projectors to overlay an intelligent environment with real-time visualizations of actions occurring in this environment (e.g., lights that are turned on or off based on the presence of someone in the room). The Feedforward Torch serves a similar goal, but requires less infrastructure and allows users to control the object they re- 
quire information about and when they need this information. Although we do not specifically focus on intelligent environments, we believe that showing feedforward through a mobile projector would also be useful for those environments.

\section{The Feedforward Torch}

The Feedforward Torch allows users to point at objects in their environment and reveal feedforward information about them, as if they were located under a spotlight. Users are shown under which conditions actions associated with the object will be executed by the system (e.g., a displacement in time or space), so that they can anticipate and adapt their behavior, if necessary. Animations are used to better convey the effect an action will have. The Feedforward Torch does not provide additional data for a physical object nor does it extend the features of a legacy system, its sole focus is on guiding the user to use the actual system.

The Feedforward Torch allows to project feedforward information on and around the system. Fig. 2 shows the Feedforward Torch prototype, consisting of a Samsung Galaxy S smart phone, a MicroVision $\mathrm{SHOWWX}^{+}$laser pico projector and a laser pointer to be able to point the device at physical objects. A custom casing was made in order to support one-handed interaction.

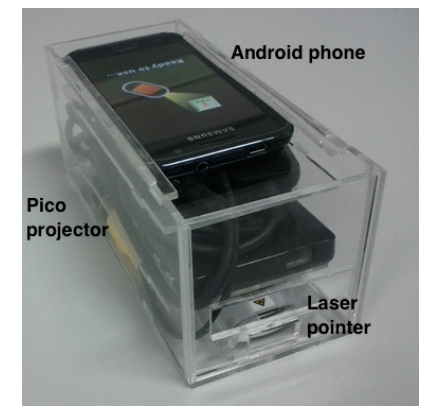

Fig. 2. The Feedforward Torch prototype

The Feedforward Torch provides an execution environment for various feedforward interfaces. The software can load configuration files describing (a) the environment, (b) the legacy systems within that environment, (c) available feedforward information for these systems and (d) properties that influence the type of presentation of the feedforward information. An example of the latter is whether the effect of an action happens after a delay and/or whether the effect will take place over a longer period of time. When the effect of an action will take place over a longer period of time the system will look for available animations that can communicate this to the user. A nice example of this can be found in Sect. 4 , in Scenario 3 "the auditorium" where one has to lower the projection screen. 
The combination of a mobile phone allows to offer both in- and out-of-context feedforward. For using the projection capabilities, the user needs to be co-located with the system and the system should be in the visual periphery of the user. But if the system is outside of the user's visual periphery, the screen of the mobile device can be used for presenting the feedforward information. The former implies the feedforward information is displayed "in-context", while using the screen of the mobile device implies some parts of the system context is lost. Feedforward information on the screen of the mobile device is also important when using projection would not be suitable (e.g., outdoors).

\section{User Study}

\subsection{Method}

We conducted a small user study to assess (1) whether the feedforward torch allows users to better understand how to work with complex legacy systems and (2) whether visualizations and animations are preferred over textual descriptions.

Since we did not implement object recognition or 3D tracking, we used a Wizard-of-Oz approach to trigger the feedforward display. Fig. 3 shows the Wizard-of-Oz setup. The wizard is standing in the background to observe the participant and used a smartphone to control the Feedforward Torch. The wizard can use the smartphone UI to select the legacy system the participant is currently pointing at from a list of supported systems.

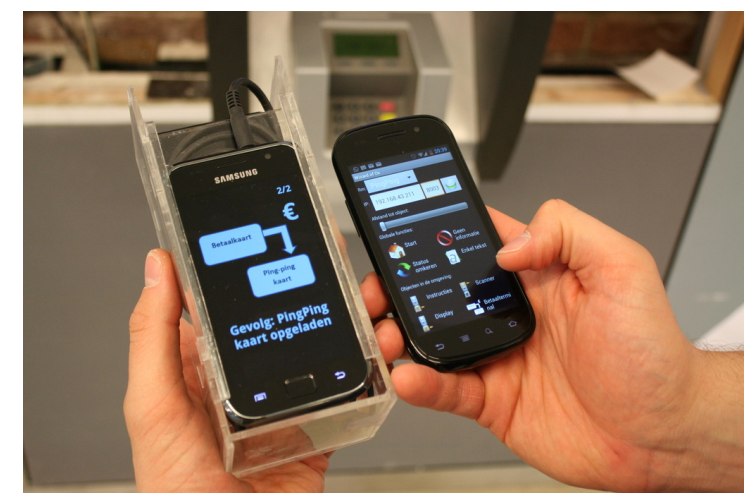

Fig. 3. The setup used for the Wizard-of-Oz study: the Feedforward Torch on the left and the control interface on the right.

The Feedforward Torch was used by 7 participants ( 5 male, 2 female; 4 without and 3 with a technical background; ages ranged from 28 to $40, \mu=32.14$ ) in three different scenarios (Fig. 4): 
- Scenario 1: "The television and the timer". Participants were asked to turn on the TV in the room, and had to work around the timer that controlled the TV.

- Scenario 2: "The PingPing NFC terminal". Participants were instructed to recharge their PingPing NFC card for the amount of 10 EUR. To do so, they had to use both their debit bank card as well as their PingPing card.

- Scenario 3: "The auditorium". In this scenario, the objective was to prepare the auditorium for a presentation. This means the projector should be turned on, the projection screen should be lowered, and the lights should be dimmed.

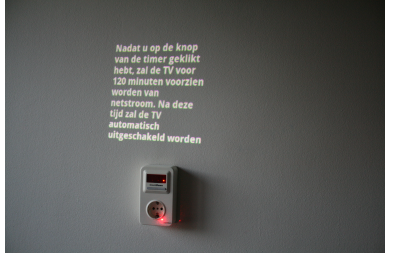

(a) Scenario 1

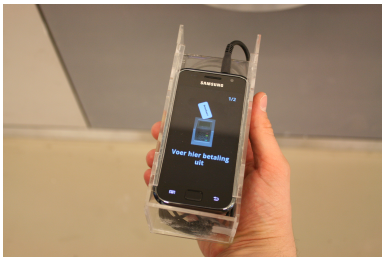

(b) Scenario 2

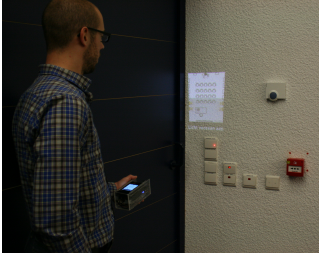

(c) Scenario 3

Fig. 4. The three scenarios used for the study.

After a short introduction of the Feedforward Torch, participants were given a specific goal they had to achieve in each of the three scenarios (e.g., turning on the TV). Each scenario took place in a different location. None of the participants were familiar with the different devices used in these scenarios.

Before participants started to explore how to complete the predefined goal, they were asked to describe to the observers how they thought the devices should be used for this purpose. Their assessment of the system was only based on its appearance and labels or signs already present in the physical space. Next, the participant used the Feedforward Torch to complete the assigned task.

When participants had performed the three tasks, we conducted semi-structured interviews in which we inquired them about the usefulness of the Feedforward Torch, and their preferences with respect to visualizations versus textual explanations. Moreover, they were asked in which situations mobile projection or the phone display was preferred.

\subsection{Results}

The Feedforward Torch helps users deal with complex situations All participants were able to complete the tasks using the Feedforward Torch. When asked about its usefulness, all participants mentioned they found the Feedforward Torch useful as a guide for complex situations. Several participants mentioned they would have been unable to complete the three scenarios without the Feedforward Torch 
or additional help from the experimenters. Two participants stated that the system would have come in handy when using the metro in a large city such as Paris or London: "When I had to use the London Underground for the first time, it would have been useful to have a device like the Feedforward Torch to help me figure out how to use the ticketing machine. Now, I had to observe other passengers first before I knew how the system worked and what I had to do."

Visualizations were preferred over textual descriptions Participants strongly preferred visualizations over textual explanations in the encountered scenarios, as they considered reading textual information to be more time-consuming. However, a number of users suggested providing detailed textual descriptions as an secondary source of information to complement the existing visualizations. As observed by Palmiter et al. [5], textual help may allow users to remember instructions more efficiently than demonstrations.

Animations are deemed useful in complex situations Especially when the result of a certain action would happen over time or outside the user's periphery, participants appreciated the use of animations. During the user study, we used for example an animation of the projection screen coming down when the participants pressed the corresponding button.

Acceptance of Mobile Projection The study revealed both advantages and disadvantages of mobile projection technology. Participants liked the fact that information was overlayed on the physical environment, so they did not have to switch between the phone display and the device they had to operate. One of the advantages of mobile projection that was mentioned during the semi-structured interviews was the fact that groups of people could explore the projection together. However, this could also cause privacy problems, in line with findings by Raskar et al. [8] and Holleis and Rukzio [9]. Another disadvantage was the difficulty of using mobile projection in low-lighting conditions. There was no clear preference for mobile projection, although we do expect hardware advancements to further improve the user experience. Based on these results, we feel that using an augmented reality approach for showing feedforward information is valuable. Although we currently use a mobile projector for this purpose, other technologies such as wearable devices (e.g., Google's Project Glass ${ }^{1}$ ) are also possible.

\section{Discussion}

We have presented the Feedforward Torch which overlays objects in a physical environment with feedforward information using a mobile projector. Based on a first user study, we feel the Feedforward Torch can help users to interact with complex devices in their environments and overcome design flaws in legacy systems. We feel this work opens up interesting avenues for further research.

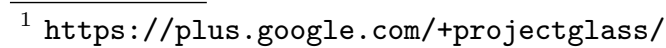


First, our current prototype is not a fully working system, but is implemented using the Wizard-of-Oz technique because we were mainly interested in exploring whether users would find the Feedforward Torch helpful. A fully working implementation would need to be able to recognize different objects of interest in the environment. Existing systems have used a combination of a projector and camera to know what the user is pointing at $[8,10]$, although the use of technologies such as QR codes or NFC tags could also prove to be useful.

Secondly, we believe it would be interesting to empower users to create feedforward information for the different objects in their environments themselves. This is similar to how people tend to augment complex devices with instructions written on labels or post-its. The success of websites such as instructables.com ${ }^{2}$ could suggest that users might be willing to do this. The Feedforward Torch could then reveal these user-made feedforward elements when pointed at the corresponding object. Finally, a larger study would need to be performed in order to provide conclusive results on the suitability of the way users interact with the Feedforward Torch.

\section{Acknowledgements}

We warmly thank Gert Vos for building the prototype of the Feedforward Torch and conducting the user study described in this paper in the context of his Master's thesis.

\section{References}

1. Olivier Bau and Wendy E. Mackay. OctoPocus: a dynamic guide for learning gesture-based command sets. In Proc. UIST '08, pages 37-46. ACM, 2008.

2. Victoria Bellotti and W. Keith Edwards. Intelligibility and accountability: human considerations in context-aware systems. Hum.-Comput. Interact., 16(2):193-212, 2001.

3. Brian Y. Lim and Anind K. Dey. Toolkit to support intelligibility in context-aware applications. In Proc. Ubicomp '10, Ubicomp '10, pages 13-22. ACM, 2010.

4. Donald A. Norman. The Design of Everyday Things. Basic Books, 2002.

5. Susan Palmiter and Jay Elkerton. Animated demonstrations for learning procedural computer-based tasks. Hum.-Comput. Interact., 8(3):193-216, 1993.

6. Claudio S. Pinhanez. The everywhere displays projector: A device to create ubiquitous graphical interfaces. In Proc. UbiComp '01, pages 315-331. Springer-Verlag, 2001.

7. Ramesh Raskar, Paul Beardsley, Jeroen van Baar, Yao Wang, Paul Dietz, Johnny Lee, Darren Leigh, and Thomas Willwacher. Rfig lamps: interacting with a selfdescribing world via photosensing wireless tags and projectors. In SIGGRAPH '05 Courses. ACM, 2005.

8. Ramesh Raskar, Jeroen van Baar, Paul Beardsley, Thomas Willwacher, Srinivas Rao, and Clifton Forlines. ilamps: geometrically aware and self-configuring projectors. In Proc. SIGGRAPH '03, pages 809-818. ACM, 2003.

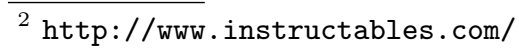


9. Enrico Rukzio and Paul Holleis. Projector phone interactions: Design space and survey. In PPD '10 Workshop, 2010.

10. Enrico Rukzio, Paul Holleis, and Hans Gellersen. Personal projectors for pervasive computing. IEEE Pervasive Computing, 11:30-37, 2012.

11. Jo Vermeulen, Jonathan Slenders, Kris Luyten, and Karin Coninx. I bet you look good on the wall: Making the invisible computer visible. In Proc. AmI '09, pages 196-205. Springer-Verlag, 2009. 